\title{
Solution of Maintenance Strategy Selection Problem by using modified Fuzzy TOPSIS for of Material Handling Equipment
}

\author{
Alia Kausar', Sana Akram¹, Muhammad Farhan Tabassum², Adeel Ahmad', \\ Shahzaman Khan ${ }^{4}$
}

\begin{abstract}
:
In today's competitive production environment, maintenance is one of the most important issues. Today in manufacturing plants productive methods are used to improve operating capacities that change environmental factors that lead to a competitive market. There is thus an important role to play in achieving those goals in selecting and explaining an optimal maintenance plan. Furthermore, there is a lack of an integrated model consisting of available requirements and choices, a systematic approach to maintenance instructions and strong maintenance decisions. In this paper modified Fuzzy TOPSIS method has been used for the solution of maintenance strategy selection problem. Linguistic variable and triangular fuzzy number have been used for modification in multi-criteria decision-making to solve maintenance strategy selection problem. Five experts have been considered for six types of maintenance strategy and ten decision criteria have been used in this problem. In this paper breakdown maintenance strategy best one out of all maintenance strategy for material handling equipment.
\end{abstract}

Keywords: Maintenance strategy selection; Multi-criteria decision-making; Fuzzy TOPSIS method; Triangular fuzzy number; Linguistic variables.

\section{Introduction}

Proper plant maintenance will significantly reduce total operating costs while increasing plant profitability. In order to provide maintenance personnel with improved technological and management skills [1], new technology and management experience must be emerging. In many companies, there is a strong motivation to optimize their equipment and plants ' life. This means that plants and machinery can go further than their original design life. Damage and efficiency analysis has therefore recently become a critical tool to improve maintenance strategy, ensure safety and reduce costs [2].

Maintenance is an unavoidable cause of costs for many businesses. The maintenance function of these companies is corrective and is carried out only under emergency conditions. In view of certain key aspects such as product quality, protection of installations

${ }^{1}$ Department of Mathematics, Lahore Garrison University, Lahore, Pakistan.

${ }^{2}$ Department of Sports Sciences \& Physical Education, Faculty of Allied Health, University of Lahore, Lahore, Pakistan.

${ }^{3}$ Department of Mathematics, Lahore Garrison University, Lahore, Pakistan.

${ }^{4}$ Department of Physical Education and Sports Sciences, Sukkur IBA University, Sukkur, Pakistan. 
and rises in maintenance costs between $15 \%$ and $70 \%$ of total cost of production, this type of interference can now be no longer accepted. [3]. Many plants have different reliability, risk, and failure criteria for different machines. It is therefore evident that different maintenance strategies for different machines must be defined in a proper maintenance program. This makes it possible to preserve production plants ' reliability and availability at an acceptable level and to prevent unnecessary investments to implement an improper maintenance strategy [4].

In the evaluation of related factors the problem of choosing maintenance approaches is a multi-criteria decision-making (MCDM). A number of solutions were proposed using fuzzy principles to solve this problem. The paper suggests a new maintenance strategy that can be the most effective maintenance strategies, depending on the level of uncertainty and also the nature and importance of the maintenance requirements [5].

Of fact, making the best possible alternative is the way to make choices. In some cases, decision-making problems are the main problem given other parameters. TOPSIS describes ideal solutions as positive and ideal solutions as negative. For the cost criteria and for the benefit criteria the positive solution is minimum and the negative ideal solution is minimum for benefit criteria and maximum for cost criteria [4]. In other types, the best possible values for the criteria consist of the optimal solution and all the worst values for the acquisition of the criteria are negative ideal solutions. As TOPSIS is an effective MCDM tool, a number of researchers have already used TOPSIS to solve many decision-making issues. Some of them de-fuzzy scores and weight in crisp values, other information has been lost during de-fuzzification. [6-8].

For the positive, ideal solution and the ideal negative solution based on criteria, Chen developed normalized values [9]. Standardized values are always $(0,0,0)$ and $(1,1,1)$ respectively for the ideal negative solution and ideal positive solution on parameters. $(0,0,0)$ and $(1,1,1)$ are extraordinary values that could be a long way from true min and high values, so that TOPSIS ' maximum and minimum estimates were not capable of extraordinary values. In Chen's work weight criteria are classified as triangular fuzzy numbers and triangular weight fuzzy numbers depending on loss of unusual values [5]. Chan's estimation is therefore very simple but incorrect triangle fuzzy numbers cannot be expressed. We have appointed fuzzy TOPSIS for the decisionmaking process of several criteria for a fluffy environment in order to avoid these problems [10].

The Fuzzy Technique for Order Preference by Similarity to Ideal Solution method (FTOPSIS) for the evaluation of maintenance strategies is used by some modification, In FTOPSIS Triangular Fuzzy Number (TFN) is used to model the uncertainty in the selection and the maintenance strategy selection problem is based on a fuzzy linguistic approach [11].

The remaining of this paper is organized as follows: in Section 2, the comprehensive detail of linguistic variable, fuzzy sets, and fuzzy numbers. In Section 3 the proposed fuzzy TOPSIS and the concepts behind it are introduced in details. In Section 4, the modified fuzzy TOPSIS ethod has been implemented for the solution of maintenance strategy selection problem. Finally, conclusions are given in Section 5.

\section{Some Basic Concepts}

Zadeh [12] first proposed the fuzzy set theory in order to deal with the vagueness of human thinking. A fuzzy collection is a class of things in which membership rates are constant. Such items are marked with a membership function that assigns a range of zero membership to one object. [12].

A linguistic variable is a variable with linguistic values. Intuitively easy-to-use linguistic terms were found to convey a 
decision-maker's subjectivity and/or conceptual inaccuracy [13].

Depending on the situation, different fuzzy numbers can be used. It is often convenient for applications to work with triangle fuzzy number (TFN) and useful for promoting the representation and processing of information in a sophisticated environment because of its computational simplicity [14].

Definition 1. If all the decision makers are the highest in terms of performance of an alternative with respect to certain $c_{n}$ parameters, then the alternative is the GPIS criteria $c_{n}$, named $G^{+}$.

Definition 2. If all the decision makers are the lowest in terms of performance of an alternative with respect to certain $c_{n}$ parameters, then the alternative is the GPIS criteria $c_{n}$, named $G^{-}$.

In many MCDM implementations, such as supplier evaluation and selection, group decision-making and performance analysis, Fuzzy TOPSIS has been described as a dominant method [15]. Junior et al. (2014) conducted an analysis of the merits of the two techniques for MCDM problems between the fuzzy TOPSIS and the fuzzy analytical hierarchy (AHP) and found Fuzzy TOPSIS is the best way to find an ideal solution [16]. It can also be used to simplify the selection process and resolve ambiguities and uncertainty. Therefore, for a multi-criteria group decision-making scenario, we described Fuzzy TOPSIS process.

In this study TFNs are adopted in the fuzzy TOPSIS methods.

\section{Proposed Fuzzy TOPSIS Method}

Through MAX and MIN operations, we can find the positive ideal and negative ideal solutions, though against the criteria of positive ideal and negative ideal solution, this fuzzy number can be picked up by $M A X$ and $M I N$ operations which have been conceivable alternative cannot be found on rating. In this paper, another generally selected TOPSIS that replaces $U_{p}$ and $L_{o}$ operations for $M A X$ and MIN operations. By $U_{p}$ and $L_{o}$ operations, a set of fuzzy numbers is ranked quickly. On this occasion, we effectively find the ideal solution and negative ideal solution, and the fuzzy number can be found extra on these conceivable alternatives against the criteria of the positive ideal and negative ideal solution.

The various steps of Fuzzy TOPSIS method are presented as follows:

STEP 1: Firstly, performance rating and weight are estimated with verbal terms. It represents the performances under linguistics classification, standardized by specialists, are: very low (VL),

low $(L)$,

medium low $(M L)$,

medium $(M)$,

medium high $(M H)$,

high $(H)$ and

very high $(\mathrm{VH})$

Choosing committee of experts for decisionmaking. $\left(E_{k} ; k=1,2 \ldots n\right)$ and then to alternative $M_{i}$ against choosing the criteria $\left(C_{j} ; j=1,2 \ldots m\right) \quad$ where $\quad G_{i j k}=$ $\left(g_{1 i j k}, g_{2 i j k}, g_{3 i j k}\right)$ is a triangular fuzzy number.

STEP 2: By using extension principle find the average performance rating of alternative $M_{i}$ against criterion $c_{j}$, is $G_{i j}$ as

$G_{i j k}=\left(g_{1 i j k}, g_{2 i j k}, g_{3 i j k}\right)$

$=1 / p \times\left(G_{i j 1}+G_{i j 2}+\cdots+G_{i j p}\right)$

By the extension principle, we have

$g_{1 i j}=\sum_{k=1}^{p} \frac{g_{1 i j k}}{p}$

$g_{2 i j}=\sum_{k=1}^{p} \frac{g_{2 i j k}}{p}$

$g_{3 i j}=\sum_{k=1}^{p} \frac{g_{3 i j k}}{p}$

STEP 3: The performance ratings of alternative $M_{1}, M_{2}, M_{3} \ldots$ composed a decision matrix that is, $G=\left[G_{i j}\right]_{m X n},\left[G_{i 1}, G_{i 2}, \ldots G_{i n}\right]$ are the performance ratings of alternative $M_{i}$. 
Let $M^{-}$and $M^{+}$are the negative ideal solution and positive ideal solution respectively;

Thus

$M^{-}=\left[G_{1}^{-}, G_{2}^{-}, \ldots G_{n}^{-}\right]$and

$M^{+}=\left[G_{1}^{+}, G_{2}^{+}, \ldots G_{n}^{+}\right]$

where $G_{j}^{-}=L_{o}\left[G_{i 1}, G_{i 2}, \ldots G_{i m}\right]$ and $G_{j}^{+}=$

$U_{p}\left[G_{i 1}, G_{i 2}, \ldots G_{i m}\right]$ for $j=1,2, \ldots, n$.

STEP 4: Next find the distance from alternatives to the negative ideal solution (or positive ideal solution). Let $d_{i j}^{-}$and $d_{i j}^{+}$be the distance from $G_{i j}$ to $G_{j}^{-}$and $G_{j}^{+}$respectively; where $i=1,2, \ldots, m ; j=1,2, \ldots, n$. Let $A=\left(a_{1}, a_{2}, a_{3}\right)$ and $B=\left(b_{1}, b_{2}, b_{3}\right)$ be two triangular fuzzy numbers. So by the definition of distance,

$d(A, B)=$

$\sqrt{\frac{1}{3}\left[\left(a_{1}-b_{1}\right)^{2}+\left(a_{2}-b_{2}\right)^{2}+\left(a_{3}-b_{3}\right)^{2}\right.}$

STEP 5: Let $W_{j k}=\left(w_{1 j k}, w_{2 j k}, w_{3 j k}\right)$ are weight evaluated by expert and $E_{k}$ under criterion $C_{j}$, where $j=1,2, \ldots, n ; k=$ $1,2, \ldots, p$

Suppose $W_{j}$ be the average weight on criterion $C_{j}$ is

$$
W_{j}=\left(w_{1 j}, w_{2 j}, w_{3 j}\right)
$$$$
=1 / p \times\left(W_{j 1}+W_{j 2}+\cdots+W_{j p}\right)
$$

where $j=1,2, \ldots, n$.

From the extension principle,

$w_{1 j}=\sum_{k=1}^{p} \frac{w_{1 j k}}{p}$
$w_{2 j}=\sum_{k=1}^{p} \frac{w_{2 j k}}{p}$
$w_{3 j}=\sum_{k=1}^{p} \frac{w_{3 j k}}{p}$

STEP 6: The weight distance of alternative $M_{i}$ to negative ideal solution $M^{-}$and ideal solution $M^{+}$respectively are find $D_{i}^{-}$and $D_{i}^{+}$. $D_{i}^{-}=\sum_{j=1}^{n} W_{j} \times d_{i j}^{-}$ and

$D_{i}^{+}=\sum_{j=1}^{n} W_{j} \times d_{i j}^{+}$,

where $i=1,2, \ldots, m$.
STEP 7: Weighted distance of $M_{i}$ can be find by $\left[D_{i}^{-}, D_{i}^{+}\right]$;

$L D^{-}=\operatorname{Lo}\left(\left\{D_{1}^{-}, D_{2}^{-}, \ldots D_{m}^{-}\right\}\right)$

$U D^{-}=U p\left(\left\{D_{1}^{-}, D_{2}^{-}, \ldots D_{m}^{-}\right\}\right)$

$L D^{+}=\operatorname{Lo}\left(\left\{D_{1}^{+}, D_{2}^{+}, \ldots D_{m}^{+}\right\}\right)$

$U D^{+}=U p\left(\left\{D_{1}^{+}, D_{2}^{+}, \ldots D_{m}^{+}\right\}\right)$

STEP 8: From these two operations Lo and $\mathrm{Up}$, the negative ideal solution is $\left[L D^{-}, U D^{+}\right]$ and the ideal solution is $\left[U D^{-}, L D^{+}\right]$find for weighted distance of all alternatives.

STEP 9: Let $M_{i}^{-}$are the distance from [ $\left.D_{i}^{-}, D_{i}^{+}\right]$to $\left[L D^{-}, U D^{+}\right]$, and $M_{i}^{+}$denote the distance from $\left[D_{i}^{-}, D_{i}^{+}\right]$to $\left[U D^{-}, L D^{+}\right]$.

Define

$M_{i}^{-}=d\left(D_{i}^{-}, L D^{-}\right)+d\left(D_{i}^{+}, U D^{+}\right)$

And

$M_{i}^{+}=d\left(D_{i}^{-}, U D^{-}\right)+d\left(D_{i}^{+}, L D^{+}\right)$

where $i=1,2, \ldots, m$.

STEP 10: Closeness coefficient $M_{i}^{*}$ of alternative $A_{i}$ is defined as:

$M_{i}^{*}=\frac{M_{i}^{-}}{M_{i}^{-}+M_{i}^{+}}$

where $i=1,2, \ldots, m$.

If $M_{i}^{*}=0$, alternative $M_{i}$ will be the poorest.

And $M_{i}^{*}=1 M_{i}$ is the best alternative.

\section{Maintenance Strategy Selection Problem for Material Handling Equipment}

In this problem their five experts, such as:

$E_{1}=$ Expert 1

$E_{2}=$ Expert 2

$E_{3}=$ Expert 3

$E_{4}=$ Expert 4

$E_{5}=$ Expert 5

And six maintenance strategies:

$M_{1}=$ corrective maintenance,

$M_{2}=$ preventive maintenance,

$M_{3}=$ condition based maintenance

$M_{4}=$ opportunistic maintenance

$M_{5}=$ predictive maintenance

$M_{6}=$ breakdown Maintenance 
Alia Kausar (et al.), Solution of Maintenance Strategy Selection Problem by using modified Fuzzy TOPSIS for of

Also this problem includes 10 evaluation criteria, such as:

$C_{1}=$ quality

$C_{2}=$ spare parts inventories

$C_{3}=$ purchasing cost of spare parts

$C_{4}=$ maintenance labour cost

$C_{5}=$ reliability

$C_{6}=$ safety

$C_{7}=$ maintenance time

$C_{8}=$ facilities

$C_{9}=$ cost of supporting equipment

$C_{10}=$ environment

The different weights of priority of each criterion and strategy are calculated using the fuzzy TOPSIS method following the construction of the hierarchy. Linguistic variables are represented in Table 1.

Table I. Fuzzy numbers and corresponding linguistic variables

\begin{tabular}{|l|c|}
\hline \multicolumn{1}{|c|}{ Linguistic Variables } & Fuzzy Number \\
\hline Very low $($ VL) & $(0.0,0.0,0.1)$ \\
\hline Low $($ L) & $(0.0,0.1,0.3)$ \\
\hline Medium low $(M L)$ & $(0.1,0.3,0.5)$ \\
\hline Medium $(M)$ & $(0.3,0.5,0.7)$ \\
\hline Medium high $(M H)$ & $(0.5,0.7,0.9)$ \\
\hline High $(H)$ & $(0.7,0.9,1.0)$ \\
\hline Very High $(\mathrm{VH})$ & $(0.9,1.0,1.0)$ \\
\hline
\end{tabular}

Step 1: The performance ratings of the six maintenance strategies in linguistic term is presented in Table 2.

Table II. Experts have assigned the correct rating in terms of linguistic variables for each criterion

\begin{tabular}{|c|c|c|c|c|c|c|}
\hline$C_{j}$ & $M_{1}$ & $M_{2}$ & $M_{3}$ & $M_{4}$ & $M_{5}$ & $M_{6}$ \\
\hline$C_{1}$ & $H, V H, H, H, V$ & $M, M H, M, M, I$ & $H, H, V H, V H, M$ & $M M, M H, H, H, M$ & $M L, M, M H, M$ & $V H, H, V H, H$, \\
\hline$C_{2}$ & $H, M H, M, M H$ & $M, M L, M, M L$, & $L, V L, L, M L, L$ & $M H, H, H, M H$, & $L, V L, M L, M L$, & $V H, H, H, H, M$ \\
\hline$C_{3}$ & $H, M H, M, M H$ & $M L, M, M, M L$, & $M L, M, M H, M$, & $M H, H, M, M H$ & $L, M L, M, M L, 1$ & $H, H, V H, V H, A$ \\
\hline$C_{4}$ & $M L, M, H, H, M$ & $H, M, L, M L, M$ & $M, M H, M$ & $M L, M, L, I$ & $L, M L, M L, M L$ & $H, V H, V H, H, V$ \\
\hline$C_{5}$ & $H, V H, V H, H$ & $M L, M, M, M H$ & $H, H, V H, H, N$ & $M H, H, M$ & $M L, M, M H, M$ & $H, H, V H, V H, A$ \\
\hline$C_{6}$ & $H, M H, M H, H$ & $M H, M, M, M H$ & $H, H, V H, H, H$ & $H, M H, M, M, N$ & $M, M H, H, M H$ & $H, H, V H, M H$ \\
\hline$C_{7}$ & $H, M H, H, H, M$ & $M, M, M L, M L$, & $M, M L, M$ & $M, M L, M$ & $M H, M, M L, M$ & $V H, H, V H, V H$ \\
\hline$C_{8}$ & $H, V H, H, M H$, & $M H, M, M, M H$ & $V H, H, V H$ & $M, M L, L, M H$ & $H, M H, H, M H$ & $M H, H, V H, V H$ \\
\hline$C_{9}$ & $H, M, M H, M$ & $M, M L, M L, H$ & $M, M H, M H, M L$ & $M L, M L, M, M$ & $M L, L, M L, M L$ & $V H, V H, H, V H$ \\
\hline & & & & & & $V H, H, H, V H, H$ \\
\hline
\end{tabular}

Step 2: The average ratings of the six maintenance strategies is in Table 3.

Table III. Average ratings of maintenance strategies

\begin{tabular}{|c|c|c|c|c|c|c|}
\hline$C_{j .}$ & $M_{1 .}$ & $M_{2 .}$ & $M_{3 .}$ & $M_{4 .}$ & $M_{5 .}$ & $M_{6 .}$ \\
\hline$C_{1}$ & $0.78,0.94,1.0$ & $0.38,0.58,0.7$ & $0.74,0.90,0.9$ & $0.54,0.74,0.9$ & $0.34,0.54,0.7$ & $0.82,0.96,1.0$ \\
\hline$C_{2}$ & $0.54,0.74,0.4$ & $0.16,0.34,0.5$ & $0.02,0.12,0.3$ & $0.54,0.74,0.9$ & $0.02,0.16,0.3$ & $0.70,0.88,0.9$ \\
\hline$C_{3}$ & $0.54,0.74,0.9$ & $0.30,0.50,0.6$ & $0.26,0.46,0.6$ & $0.50,0.70,0.8$ & $0.16,0.34,0.5$ & $0.78,0.94,1.0$ \\
\hline$C_{4}$ & $0.42,0.62,0.7$ & $0.24,0.42,0.6$ & $0.26,0.46,0.6$ & $0.16,0.34,0.5$ & $0.12,0.30,0.5$ & $0.82,0.96,1.0$ \\
\hline$C_{5}$ & $0.74,0.90,0.9$ & $0.34,0.54,0.7$ & $0.70,0.88,0.9$ & $0.50,0.70,0.8$ & $0.26,0.46,0.6$ & $0.78,0.94,1.0$ \\
\hline$C_{6}$ & $0.58,0.78,0.9$ & $0.46,0.66,0.8$ & $0.74,0.92,1.0$ & $0.46,0.66,0.8$ & $0.46,0.66,0.8$ & $0.66,0.84,0.9$ \\
\hline$C_{7}$ & $0.62,0.82,0.9$ & $0.30,0.50,0.6$ & $0.26,0.46,0.6$ & $0.42,0.62,0.8$ & $0.20,0.38,0.5$ & $0.86,0.98,1.0$ \\
\hline$C_{8}$ & $0.66,0.84,0.9$ & $0.38,0.58,0.8$ & $0.82,0.96,1.0$ & $0.20,0.38,0.5$ & $0.58,0.78,0.9$ & $0.78,0.92,0.9$ \\
\hline$C_{9}$ & $0.46,0.66,0.8$ & $0.34,0.54,0.7$ & $0.38,0.58,0.7$ & $0.18,0.38,0.5$ & $0.12,0.30,0.5$ & $0.86,0.98,1.0$ \\
\hline
\end{tabular}


Alia Kausar (et al.), Solution of Maintenance Strategy Selection Problem by using modified Fuzzy TOPSIS for of

\begin{tabular}{|l|l|l|l|l|l|l|}
\hline$C_{10}$ & $0.62,0.80,0.9$ & $0.62,0.82,0.9$ & $0.82,0.96,1.0$ & $0.46,0.66,0.8$ & $0.62,0.82,0.9$ & $0.78,0.91,1.0$ \\
\hline
\end{tabular}

Step 3: By the performance ratings of alternative composed a decision-making matrix $G$ is in Table 4.

Table IV. Decision-making matrix

\begin{tabular}{|c|c|c|c|}
\hline$G_{1}^{+}=0.82,0.96,1.00$ & $G_{6}^{+}=0.74,0.92,1.00$ & $G_{1}^{-}=0.34,0.54,0.74$ & $G_{6}^{-}=0.46,0.66,0.84$ \\
\hline$G_{2}^{+}=0.70,0.88,0.98$ & $G_{7}^{+}=0.86,0.98,1.00$ & $G_{2}^{-}=0.02,0.12,0.30$ & $G_{7}^{-}=0.20,0.38,0.58$ \\
\hline$G_{3}^{+}=0.78,0.94,1.00$ & $G_{8}^{+}=0.82,0.96,1.00$ & $G_{3}^{-}=0.16,0.34,0.54$ & $G_{8}^{-}=0.20,0.38,0.58$ \\
\hline$G_{4}^{+}=0.82,0.96,1.00$ & $G_{9}^{+}=0.86,0.98,1.00$ & $G_{4}^{-}=0.12,0.30,0.50$ & $G_{9}^{-}=0.12,0.30,0.50$ \\
\hline$G_{5}^{+}=0.78,0.94,1.00$ & $\begin{array}{l}G_{10}^{+} \\
=0.82,0.96,1.00\end{array}$ & $G_{5}^{-}=0.26,0.46,0.66$ & $G_{10}^{-}=0.46,0.66,0.84$ \\
\hline
\end{tabular}

Step 4: The distance values for the six maintenance strategies on 10 criteria is in Table 5.

Table V. Distance values for maintenance strategies

\begin{tabular}{|c|c|c|c|c|c|c|}
\hline \multirow{2}{*}{$C_{j}$} & \multicolumn{2}{|c|}{$M_{1}$} & \multicolumn{2}{|c|}{$M_{2}$} & \multicolumn{2}{c|}{$M_{3}$} \\
\cline { 2 - 7 } & $\begin{array}{c}\mathrm{d} \\
\left(G_{1 j}, G_{j^{+}}\right)\end{array}$ & $\mathrm{d}\left(G_{1 j}, G_{j^{-}}\right)$ & $\mathrm{d}\left(G_{2 j}, G_{j^{+}}\right)$ & $\mathrm{d}\left(G_{2 j}, G_{j^{-}}\right)$ & $\mathrm{d}\left(G_{3 j}, G_{j^{+}}\right)$ & $\mathrm{d}\left(G_{3 j}, G_{j^{-}}\right)$ \\
\hline$C_{1}$ & 0.08246 & 0.3747 & 0.3589 & 0.0400 & 0.0589 & 0.3402 \\
\hline$C_{2}$ & 0.1311 & 0.5816 & 0.5089 & 0.2046 & 0.7077 & 0.0000 \\
\hline$C_{3 .}$ & 0.1894 & 0.3804 & 0.4189 & 0.1469 & 0.4533 & 0.1137 \\
\hline$C_{4}$ & 0.3286 & 0.3004 & 0.5125 & 0.1137 & 0.4758 & 0.1536 \\
\hline$C_{5}$ & 0.0346 & 0.4189 & 0.3747 & 0.0800 & 0.0589 & 0.3968 \\
\hline$C_{6}$ & 0.1275 & 0.1137 & 0.2392 & 0.0000 & 0.0000 & 0.2392 \\
\hline$C_{7}$ & 0.1681 & 0.4141 & 0.4642 & 0.1071 & 0.5033 & 0.0673 \\
\hline$C_{8}$ & 0.1178 & 0.4350 & 0.3514 & 0.2082 & 0.0000 & 0.5469 \\
\hline$C_{9}$ & 0.3098 & 0.3468 & 0.4252 & 0.2269 & 0.03824 & 0.2735 \\
\hline$C_{10}$ & 0.1549 & 0.1311 & 0.1428 & 0.1479 & 0.0000 & 0.2859 \\
\hline$C_{j}$ & & & & & $M_{6}$ & $M_{6}$ \\
\cline { 2 - 7 } & $\mathrm{d}\left(G_{4 j}, G_{j}\right)$ & $\mathrm{d}\left(G_{4 j}, G_{j}-\right)$ & $\mathrm{d}\left(G_{5 j}, G_{j^{+}}\right)$ & $\mathrm{d}\left(G_{5 j}, G_{j}-\right)$ & $\mathrm{d}\left(G_{6 j}, G_{j^{+}}\right)$ & $\mathrm{d}\left(G_{6 j}, G_{j^{-}}\right)$ \\
\hline$C_{1}$ & 0.2135 & 0.1876 & 0.3977 & 0.0000 & 0.0000 & 0.3977 \\
\hline$C_{2}$ & 0.1311 & 0.5816 & 0.6808 & 0.0327 & 0.0000 & 0.7077 \\
\hline$C_{3}$ & 0.2239 & 0.3468 & 0.5645 & 0.0000 & 0.0000 & 0.5540 \\
\hline$C_{4}$ & 0.5864 & 0.0400 & 0.6260 & 0.0000 & 0.0000 & 0.6260 \\
\hline$C_{5}$ & 0.2277 & 0.2274 & 0.4533 & 0.0000 & 0.0000 & 0.4532 \\
\hline$C_{6}$ & 0.2392 & 0.0000 & 0.2392 & 0.0000 & 0.0693 & 0.1701 \\
\hline$C_{7}$ & 0.3479 & 0.2269 & 0.5692 & 0.0000 & 0.0000 & 0.5692 \\
\hline$C_{8}$ & 0.5469 & 0.0000 & 0.1736 & 0.3934 & 0.0346 & 0.5125 \\
\hline$C_{9}$ & 0.5770 & 0.7394 & 0.6481 & 0.0000 & 0.0000 & 0.6481 \\
\hline$C_{10}$ & 0.2859 & 0.0000 & 0.1428 & 0.1479 & 0.0258 & 0.2622 \\
\hline
\end{tabular}


Step 5: The linguistic weights and average ratings for 10 criteria are in Table 6-7.

Table VI. Linguistic weights for criteria

\begin{tabular}{|c|c|c|c|c|c|}
\hline$C_{j}$ & $E_{1}$ & $E_{2}$ & $E_{3}$ & $E_{4}$ & $E_{5}$ \\
\hline$C_{1}$ & $V H$ & $H$ & $V H$ & $V H$ & $H$ \\
\hline$C_{2}$ & $M H$ & $M$ & $M L$ & $L$ & $V L$ \\
\hline$C_{3}$ & $M$ & $M L$ & $M$ & $M H$ & $M L$ \\
\hline$C_{4}$ & $M H$ & $H$ & $M L$ & $M$ & $M$ \\
\hline$C_{5}$ & $V H$ & $H$ & $H$ & $V H$ & $V H$ \\
\hline$C_{6}$ & $H$ & $V H$ & $H$ & $V H$ & $V H$ \\
\hline$C_{7}$ & $M H$ & $M$ & $H$ & $M H$ & $H$ \\
\hline$C_{8}$ & $H$ & $M H$ & $V H$ & $H$ & $M H$ \\
\hline$C_{9}$ & $L$ & $M L$ & $M$ & $M$ & $V L$ \\
\hline$C_{10}$ & $M H$ & $H$ & $V H$ & $H$ & $M H$ \\
\hline
\end{tabular}

Table VII. Average ratings

\begin{tabular}{|c|c|c|c|c|c|}
\hline$C_{j}$ & $E_{1}$ & $E_{2}$ & $E_{3}$ & $E_{4}$ & $E_{5}$ \\
\hline$C_{1}$ & $0.9,1.0,1.0$ & $0.7,0.9,1.0$ & $0.9,1.0,1.0$ & $0.9,1.0,1.0$ & $0.7,0.9,1.0$ \\
\hline$C_{2}$ & $0.5,0.7,0.9$ & $0.3,0.5,0.7$ & $0.1,0.3,0.5$ & $0.0,0.1,0.3$ & $0.0,0.0,0.1$ \\
\hline$C_{3}$ & $0.3,0.5,0.7$ & $0.1,0.3,0.5$ & $0.3,0.5,0.7$ & $0.5,0.7,0.9$ & $0.1,0.3,0.5$ \\
\hline$C_{4}$ & $0.5,0.7,0.9$ & $0.7,0.9,1.0$ & $0.1,0.3,0.5$ & $0.3,0.5,0.7$ & $0.3,0.5,0.7$ \\
\hline$C_{5}$ & $0.9,1.0,1.0$ & $0.7,0.9,1.0$ & $0.7,0.9,1.0$ & $0.9,1.0,1.0$ & $0.9,1.0,1.0$ \\
\hline$C_{6}$ & $0.7,0.9,1.0$ & $0.9,1.0,1.0$ & $0.7,0.9,1.0$ & $0.9,1.0,1.0$ & $0.9,1.0,1.0$ \\
\hline$C_{7}$ & $0.5,0.7,0.9$ & $0.3,0.5,0.7$ & $0.7,0.9,1.0$ & $0.5,0.7,0.9$ & $0.7,0.9,1.0$ \\
\hline$C_{8}$ & $0.7,0.9,1.0$ & $0.5,0.7,0.9$ & $0.9,1.0,1.0$ & $0.7,0.9,1.0$ & $0.5,0.7,0.9$ \\
\hline$C_{9}$ & $0.0,0.1,0.3$ & $0.1,0.3,0.5$ & $0.3,0.5,0.7$ & $0.3,0.5,0.7$ & $0.0,0.0,0.1$ \\
\hline$C_{10}$ & $0.5,0.7,0.9$ & $0.7,0.9,1.0$ & $0.9,1.0,1.0$ & $0.7,0.9,1.0$ & $0.5,0.7,0.9$ \\
\hline
\end{tabular}

Calculated average weights against the 10 criteria from Table 7 are:

$$
\begin{aligned}
& W_{1}=(0.82,0.96,1.00) \\
& W_{2}=(0.18,0.32,0.50) \\
& W_{3}=(0.26,0.46,0.66) \\
& W_{4}=(0.38,0.58,0.76) \\
& W_{5}=(0.82,0.96,1.00) \\
& W_{6}=(0.82,0.96,1.00) \\
& W_{7}=(0.54,0.74,0.90) \\
& W_{8}=(0.66,0.84,0.96) \\
& W_{9}=(0.14,0.28,0.46) \\
& W_{10}=(0.66,0.84,0.96)
\end{aligned}
$$

Step 6: The weighted distance values of six maintenance strategies on 10 criteria are presented as follows.

$$
\begin{aligned}
& D_{1}^{+}=(1.3209,1.7071,1.9826) \\
& D_{2}^{+}=(1.8293,2.4644,2.9810) \\
& D_{3}^{+}=(0.8680,1.3035,1.7613) \\
& D_{4}^{+}=(1.6803,2.2561,2.7167) \\
& D_{5}^{+}=(2.0081,2.7557,3.3931) \\
& D_{6}^{+}=(0.0969,0.1175,0.1276) \\
& D_{1}^{-}=(1.7075,2.2854,2.7531) \\
& D_{2}^{-}=(0.5413,0.7561,0.9483) \\
& D_{3}^{-}=(1.5127,1.9045,2.1538) \\
& D_{4}^{-}=(0.7764,1.1422,1.5094) \\
& D_{5}^{-}=(0.4161,0.5593,0.6831)
\end{aligned}
$$


$D_{6}^{-}=(2.2559,3.0780,3.7704)$

Step 7: The values of weighted distance are given below

$L D^{+}=(0.0969,0.1175,0.1276)$

$U D^{+}=(2.0081,2.7557,3.3931)$

$L D^{-}=(0.4161,0.5593,0.6831)$

$U D^{-}=(2.2559,3.0780,3.7704)$

Step 8: Positive and negative ideal solutions from weighted distance are given below

$d\left(D_{1}^{+}, U D^{+}\right)=1.0893$

$d\left(D_{1}^{+}, L D^{+}\right)=1.5774$

$d\left(D_{2}^{+}, U D^{+}\right)=0.8378$

$d\left(D_{2}^{+}, L D^{+}\right)=2.3559$

$d\left(D_{3}^{+}, U D^{+}\right)=1.4224$

$d\left(D_{3}^{+}, L D^{+}\right)=1.2477$

$d\left(D_{4}^{+}, U D^{+}\right)=0.5209$

$d\left(D_{4}^{+}, L D^{+}\right)=2.1435$

$d\left(D_{5}^{+}, U D^{+}\right)=0.0000$

$d\left(D_{5}^{+}, L D^{+}\right)=2.6629$

$d\left(D_{6}^{+}, U D^{+}\right)=2.6629$

$d\left(D_{6}^{+}, L D^{+}\right)=0.0000$

and

$d\left(D_{1}^{-}, U D^{-}\right)=0.8091$

$d\left(D_{1}^{-}, L D^{-}\right)=1.7255$

$d\left(D_{2}^{-}, U D^{-}\right)=2.3532$

$d\left(D_{2}^{-}, L D^{-}\right)=0.2039$

$d\left(D_{3}^{-}, U D^{-}\right)=1.2306$

$d\left(D_{3}^{-}, L D^{-}\right)=1.3134$

$d\left(D_{4}^{-}, U D^{-}\right)=1.9191$

$d\left(D_{4}^{-}, L D^{-}\right)=0.6198$

$d\left(D_{5}^{-}, U D^{-}\right)=2.5338$

$d\left(D_{5}^{-}, L D^{-}\right)=0.0000$

$d\left(D_{6}^{-}, U D^{-}\right)=0.0000$

$d\left(D_{6}^{-}, L D^{-}\right)=2.5338$

Step 9: From these above distance values, $M_{i}^{+}$ and $M_{i}^{-}$can be calculated:
$M_{1}^{+}=2.3865 \quad M_{1}^{-}=2.8148$
$M_{2}^{+}=4.7091 \quad M_{2}^{-}=1.0417$
$M_{3}^{+}=2.4783 \quad M_{3}^{-}=2.7358$
$M_{4}^{+}=4.0626 \quad M_{4}^{-}=1.1407$
$M_{5}^{+}=5.1967 \quad M_{5}^{-}=0.0000$
$M_{6}^{+}=0.0000 \quad M_{6}^{-}=5.1967$

Step 10: Finally, all the results are evaluated, final Scores and ranks are given in Table 8.

$M_{1}^{*}=0.5412$

$M_{2}^{*}=0.1811$

$M_{3}^{*}=0.5247$

$M_{4}^{*}=0.2192$

$M_{5}^{*}=0.0000$

$M_{6}^{*}=1.0000$

Table VIII. Final Scores and ranks of strategies

\begin{tabular}{|c|c|c|}
\hline Strategy & Final Scores & Ranks \\
\hline$M_{1}$ & 0.5412 & 2 \\
\hline$M_{2}$ & 0.1811 & 5 \\
\hline$M_{3}$ & 0.5247 & 3 \\
\hline$M_{4}$ & 0.2192 & 4 \\
\hline$M_{5}$ & 0.0000 & 6 \\
\hline$M_{6}$ & 1.0000 & 1 \\
\hline
\end{tabular}

Clearly, from Table 8 the ranking order is $M_{6}>M_{1}>M_{3}>M_{4}>M_{2}>M_{5}$

Therefore, performances of $M_{6}$ is the best.

\section{Result and Discussion}

The result reported in [17] the same problem has been solved with the help of fuzzy SAW method the order ranking of maintenance strategy for material handling equipment. The results are as follows

$M 6>M 1>M 3>M 4>M 2>M 5$.

And from the above experimentation we have the same results which showed that breakdown maintenance $M_{6}$ is the best maintenance strategy for material handling equipment and predictive maintenance $M_{5}$ is the poor maintenance strategy for material handling equipment.

\section{Conclusion}

In this paper modified Fuzzy TOPSIS method has been used for the solution of maintenance strategy selection problem. Linguistic variable and triangular fuzzy number have been used for modification in multi-criteria decisionmaking to solve maintenance strategy selection 
problem. Five experts have been considered for six types of maintenance strategy and ten decision criteria have been used in this problem. Two operators $U_{p}$ and $L_{o}$, which satisfied fuzzy numbers of partial ordering relations for generalized of TOPSIS, these two operations are used to determine the negative ideal and positive ideal solutions in a fuzzy environment. Ultimately, it is concluded that the malfunction management approach for equipment for material handling is best of all maintenance strategies.

\section{REFERENCES}

[1] Bertolini, M. and M. Bevilacqua, A combined goal programming-AHP approach to maintenance selection problem. Reliability Engineering \& System Safety, 2006. 91(7): p. 839-848.

[2] Yatomi, M., et al., Application of risk-based maintenance on materials handling systems. IHI engineering review, 2004. 37(2): p. 52-58.

[3] Sarkar, A., D.K. Behera, and B. Sarkar, The maintenance strategy selection of a gas turbine power plant system. Journal of Information and Operations Management, 2011. 2(1): p. 9.

[4] Akhshabi, M., A new fuzzy multi criteria model for maintenance policy. Middle-East Journal of Scientific Research, 2011. 10(1): p. 33-38.

[5] Saeed, M. and M. Tabassum, COMPARISON BETWEEN FUZZY SOFT MATRIX (FSM) AND INTERVAL VALUED FUZZY SOFT MATRIX (IVFSM) IN DECISION MAKING. Science International, 2016. 28(5)

[6] Saeed, M., et al., Generalization of TOPSIS from Soft Set to Fuzzy Soft Sets in Decision Making Problem. Scientific Inquiry Review, 2017. 1(1): p. 11-18.

[7] Tsaur, S.-H., T.-Y. Chang, and C.-H. Yen, The evaluation of airline service quality by fuzzy MCDM. Tourism management, 2002. 23(2): p. 107-115.

[8] Ahmad, A., et al., Multi-Criteria DecisionMaking for Airport Operation Performance Using Triangular Fuzzy Numbers. Scientific Inquiry Review, 2019. 3(3): p. 01-15.

[9] Chen, C.-T., Extensions of the TOPSIS for group decision-making under fuzzy environment. Fuzzy sets and systems, 2000. 114(1): p. 1-9.
[10] Gani, A.N. and S.M. Assarudeen, A new operation on triangular fuzzy number for solving fuzzy linear programming problem. Applied Mathematical Sciences, 2012. 6(11): p. 525-532.

[11] Omar, M.N. and A.R. Fayek, A TOPSIS-based approach for prioritized aggregation in multicriteria decision-making problems. Journal of Multi-Criteria Decision Analysis, 2016. 23(56): p. 197-209.

[12] Zadeh, L.A., Fuzzy sets. Information and control, 1965. 8(3): p. 338-353.

[13] Zadeh, L.A., The concept of a linguistic variable and its application to approximate reasoning-III. Information sciences, 1975. 9(1): p. 43-80.

[14] Ertuğrul, İ. and N. Karakaşoğlu, Comparison of fuzzy AHP and fuzzy TOPSIS methods for facility location selection. The International Journal of Advanced Manufacturing Technology, 2008. 39(7-8): p. 783-795.

[15] Bottani, E. and A. Rizzi, A fuzzy TOPSIS methodology to support outsourcing of logistics services. Supply Chain Management: An International Journal, 2006. 11(4): p. 294308.

[16] Junior, F.R.L., L. Osiro, and L.C.R. Carpinetti, A comparison between Fuzzy AHP and Fuzzy TOPSIS methods to supplier selection. Applied Soft Computing, 2014. 21: p. 194-209.

[17] Sagara, M.K., P. Jayaswala, and K. Kushwahc, Exploring Fuzzy SAW Method for Maintenance Strategy Selection Problem of Material Handling Equipment. 2013. 